\title{
Dietary patterns in adolescents: a comparison of cluster and principal component analyses
}

\author{
Á. P. Hearty and M. J. Gibney \\ Institute of Food \& Health, School of Agriculture, Food Science and Veterinary Medicine, University College Dublin, \\ Belfield, Dublin 4, Republic of Ireland
}

Traditionally, nutrition research has been focused on the detailed examination of nutrients and dietary components. However, in more recent years public health nutrition has seen a move from research at the nutrient level to the food level. Both cluster and principal component (PC) analysis (PCA) offer a means of exploring dietary patterns at this level. In cluster analysis dietary data are reduced into mutually-exclusive patterns based on differences in dietary intakes. In PCA dietary patterns are derived based on the correlation matrix of the original food variables and individuals receive a continuous factor score for each PC. Without effective intervention poor eating habits developed during adolescence may 'track' into adulthood ${ }^{(1)}$.Thus, it is important to document their dietary practices and identify areas in which strategies can be focused. In the literature there are only a few studies on the dietary patterns of adolescents ${ }^{(2,3)}$ and there appear to be none that have compared cluster and PCA methods. The National Teens' Food Survey was conducted in the Republic of Ireland in 2005-6 on a representative sample of 441 teenagers aged 13-17 years ${ }^{(4)}$. A semi-quantitative $7 \mathrm{~d}$ food diary was used to collect information on foods and beverages consumed. Food intake data were reduced to thirty-two food groups. Food groups were expressed either as mean intake $(\mathrm{g} / \mathrm{d})$ or as the percentage contribution to total energy intake (\%TE). In order to compare the patterns derived by both methods, mean PC scores were calculated and computed across the cluster solutions.

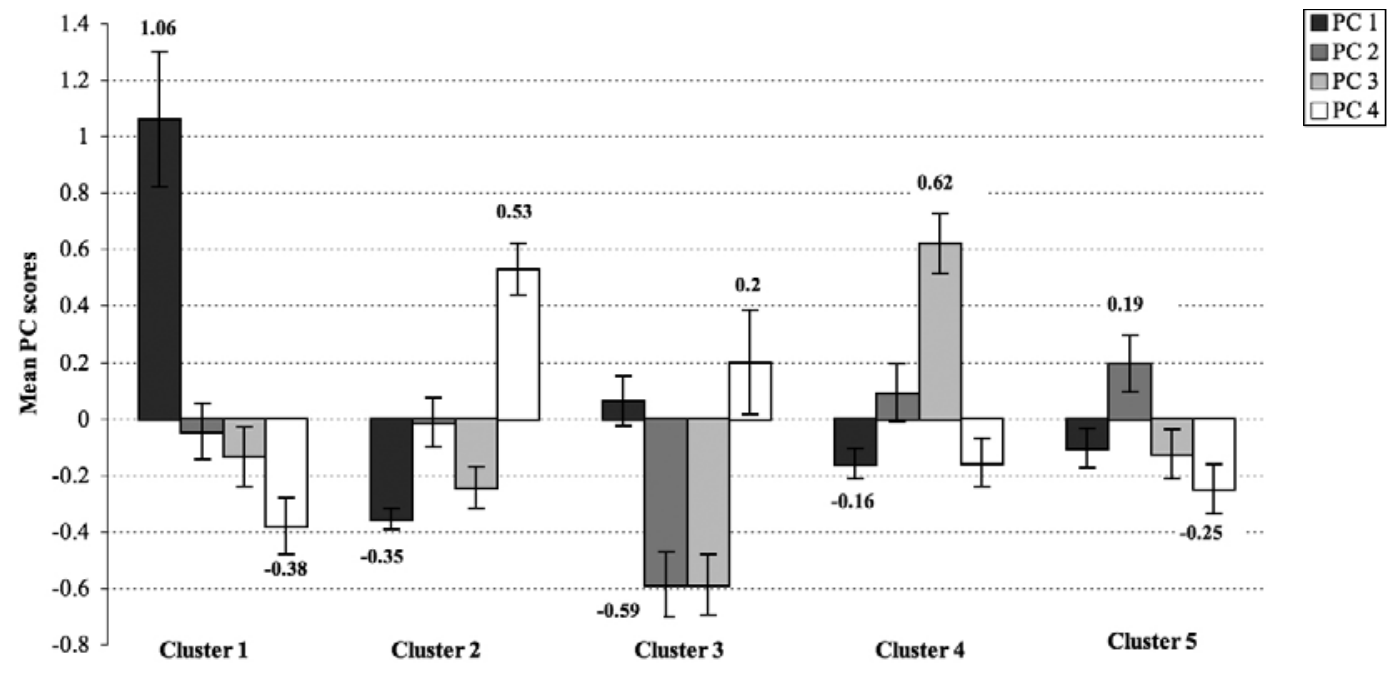

Key: Cluster 1: 'Healthy', Cluster 2: 'Unhealthy', Cluster 3: 'Rice/Pasta Dishes', Cluster 4: 'Sandwich', Cluster 5: 'Breakfast cereal \& main meal type foods' PC=Principal Component. PC 1: 'Healthy', PC 2: 'Traditional', PC 3: 'Sandwich', PC 4. 'Unhealthy'

The most sensible clusters were derived based on \%TE, which were labelled as 'Healthy', 'Unhealthy', 'Rice/pasta dishes', 'Sandwich' and 'Breakfast cereal \& main meal-type foods'. The most interpretable patterns using PCA were derived based on intake (g/d) and were labelled as 'Healthy', 'Traditional', 'Sandwich' and 'Unhealthy'. Cluster 1 scored highest for PC 1 and lowest for PC 4, indicating that close similarities exist between the 'Healthy' pattern derived by both methods, and that it is most different from the 'Unhealthy' PC. Cluster 2 scored highest for PC 4, also indicating that for the 'Unhealthy' pattern both cluster and PCA derived very similar patterns. In summary, cluster analysis and PCA, although statistically-different methods, identified similar dietary patterns when presented with the same dataset, and these patterns were directly comparable in the adolescent sample.

1. Mikkila V, Rasanen L, Raitakari OT, Pietinen P \& Viikari J (2005) Br J Nutr 93, 923-931.

2. Aranceta J, Perez-Rodrigo C, Ribas L, Serra-Majem L (2003) Eur J Clin Nutr 57 (Suppl 1), S40-44.

3. Ritchie LD, Spector P, Stevens MJ et al. (2007) J Nutr 135, 399-406.

4. Irish Universities Nutrition Alliance (2006) National Teens' Food Survey. www.iuna.net 